\title{
Quantum product and parabolic orbits in homogeneous spaces
}

\author{
Clélia Pech
}

June 21, 2013

\begin{abstract}
Chaput, Manivel and Perrin proved in [3] a formula describing the quantum product by Schubert classes associated to cominuscule weights in a rational projective homogeneous space $X$. In the case where $X$ has Picard rank one, we relate this formula to the stratification of $X$ by $P$-orbits, where $P$ is the parabolic subgroup associated to the cominuscule weight. We deduce a decomposition of the Hasse diagram of $X$, i.e the diagram describing the cup-product with the hyperplane class. For all classical Grassmannians, we give a complete description of parabolic orbits associated to cominuscule weights and we make the decomposition of the Hasse diagram explicit.
\end{abstract}

Keywords : generalized flag varieties, quantum product, parabolic orbits, Hasse diagrams MSC classes : 14M15, 14N35

\section{Introduction}

Let $G$ be a semisimple algebraic group over $\mathbb{C}, B$ be a Borel subgroup and $T \subset B$ be a maximal torus. We denote by $\Phi$ the set of roots of $G$ with respect to $T, \Phi^{+}$the subset of positive roots with respect to $B, \Delta=\left\{\alpha_{1}, \ldots, \alpha_{n}\right\}$ the subset of simple roots and $W$ the Weyl group of $G$. A fundamental weight $\omega$ is said to be minuscule if $\left|\left\langle\alpha^{\vee}, \omega\right\rangle\right| \leq 1$ for all $\alpha \in \Phi$, where $\alpha^{\vee}$ is the coroot of $\alpha$. It is said to be cominuscule if it is minuscule for the dual root system. Fundamental weights will be denoted $\omega_{1}, \ldots, \omega_{n}$, in the same order as in [1].

Let $Q \supset B$ be a parabolic subgroup of $G$ and denote by $X$ the homogeneous space $G / Q$. In [3], Chaput, Manivel and Perrin proved a formula describing the quantum product in $X$ by special Schubert classes associated to cominuscule weights. These classes correspond to the elements of the image of Seidel's representation $\pi_{1}\left(G^{\text {ad }}\right) \rightarrow \mathrm{QH}^{*}(G / Q)_{\text {loc }}^{\times}\left[11\right.$, where $G^{\text {ad }}=G / Z(G)$ and $\mathrm{QH}^{*}(G / Q)_{\text {loc }}^{\times}$is the group of invertible elements in the small quantum cohomology $\operatorname{ring} \mathrm{QH}^{*}(G / Q)$ localized in the quantum parameters. Before stating this formula, we introduce some notation for the quantum cohomology of $X$.

The (small) quantum cohomology ring $\mathrm{QH}^{*}(X)$ of a homogeneous variety $X=G / Q$ is a deformation of its cohomology ring. Consider the parameter ring

$$
\Lambda=\left\{\sum_{\beta} a_{\beta} q^{\beta} \mid \beta \in \mathrm{H}_{2}^{+}(X, \mathbb{Z}), a_{\beta} \in \mathbb{Z}\right\},
$$

where the sums are finite, $\mathrm{H}_{2}^{+}(X, \mathbb{Z})$ denotes the set of effective cycles in $H_{2}(X, \mathbb{Z})$ and the $q^{\beta}$ are formal parameters such that $q^{\beta} q^{\beta^{\prime}}=q^{\beta+\beta^{\prime}}$. As a $\mathbb{Z}$-module, the quantum cohomology $\operatorname{ring} \mathrm{QH}^{*}(X)$ is isomorphic to $\mathrm{H}^{*}(X, \mathbb{Z}) \otimes_{\mathbb{Z}} \Lambda$. Moreover, it admits a ring structure defined by the quantum product $\star$, which is a deformation of the cup-product. A precise definition for the quantum product can be found in [5]. The group $H_{2}(X, \mathbb{Z})$ contains $\Phi^{\vee} / \Phi_{Q}^{\vee}$, where $\Phi^{\vee}$ denotes the coroot lattice of $G$ and $\Phi_{Q}^{\vee}$ the coroot lattice of $Q$, hence positive coroots can be seen as effective classes $\beta \in \mathrm{H}_{2}^{+}(X, \mathbb{Z})$.

Now let $\mathcal{I}$ be the set of vertices of the Dynkin diagram of $G$ corresponding to cominuscule weights. If $i \in \mathcal{I}$, let $v_{i}$ be the shortest element of the Weyl group $W$ such that $v_{i} \omega_{i}^{\vee}=w_{0} \omega_{i}^{\vee}$, where $\omega_{i}^{\vee}$ is the fundamental coweight associated to $i$ and $w_{0}$ is the longest element of $W$. Then 
the quantum product in $X$ by the Schubert class $\sigma_{v_{i}}$ Poincaré dual to the Schubert cycle $\left[X_{v_{i} w_{0}}\right]$ is given by the following formula :

Theorem 1 ([3], Thm.1]). For all $w \in W$ and for all $i \in \mathcal{I}$, we have :

$$
\sigma_{v_{i}} \star \sigma_{w}=q^{\eta_{Q}\left(\omega_{i}^{\vee}-w^{-1}\left(\omega_{i}^{\vee}\right)\right)} \sigma_{v_{i} w},
$$

where $\eta_{Q}: \Phi^{\vee} \rightarrow \Phi^{\vee} / \Phi_{Q}^{\vee}$ is the natural surjection.

The aim of this paper is to relate the above theorem to a stratification of $X=G / Q$ by $P_{i}$-orbits when $Q$ is a maximal parabolic and $P_{i}$ is the maximal parabolic associated to the weight $\omega_{i}$. In Section 2, we recall some well-known facts about parabolic orbits and we describe the parabolic orbits associated to cominuscule weights in the classical Grassmannians. Then in Section 3 , we explain the link between Thm. 1 and the stratification by parabolic orbits in $X$. We deduce in Section 4 a decomposition of the Hasse diagram of the classical Grassmannians.

\section{Parabolic orbits}

In 2.1 we recall some classical facts about parabolic orbits in (generalized) flag varieties, and in 2.2 we give a more explicit description of parabolic orbits associated to cominuscule weights in the classical Grassmannians.

\subsection{Parabolic orbits in generalized flag varieties}

A generalized flag variety is a variety of the form $X=G / Q$, where $G$ is a semisimple algebraic group, $B$ a Borel subgroup and $Q \supset B$ a parabolic subgroup. Now consider a second parabolic subgroup $P \supset B$. We call $P$-orbits or parabolic orbits the orbits of $X$ under the action of $P$ by left multiplication. Here are some elementary properties of parabolic orbits, which can be found in [10, Sec. 2.1] :

Proposition 2. 1. Every $P$-orbit can be written as $P w Q / Q$ with $w \in W$.

2. The P-orbits are smooth and locally closed, indexed by double cosets $W_{P} \backslash W / W_{Q}$, where $W_{P}$ and $W_{Q}$ denote the Weyl groups associated to $P$ and $Q$. Moreover, they define a stratification of $X$ :

$$
X=\bigsqcup_{W_{P} w W_{Q} \in W_{P} \backslash W / W_{Q}} P w Q / Q .
$$

3. The P-orbits are B-stable, hence they are a union of Schubert cells :

$$
P w Q / Q=\bigcup_{\left(w_{P}, w_{Q}\right) \in W_{P} \times W_{Q}} B w_{P} w w_{Q} Q / Q .
$$

We denote by $W^{Q}$ the set of minimal length representatives of cosets in $W / W_{Q}$, which inherits the Bruhat order of $W$. Let us describe the double cosets indexing parabolic orbits :

Proposition 3. Let $\mathcal{E}=W_{P} w W_{Q}$ be a double coset in $W_{P} \backslash W / W_{Q}$. Then $\mathcal{E} \cap W^{Q}$ contains unique minimal and maximal elements $w_{\min }$ and $w_{\max }$. Moreover, it is equal to the interval $\left[w_{\min }, w_{\max }\right]$ for the Bruhat order in $W^{Q}$.

This statement in proved in [7. In particular, we see that parabolic orbits correspond to some sub-intervals of $W^{Q}$. The next result describes them as the total space of a vector bundle over another generalized flag variety.

First of all, consider the Levi decomposition $P=L \ltimes U$, where $L$ is a Levi subgroup and $U$ is the unipotent radical of $P$. If $\mathcal{O}$ is a $P$-orbit associated to a double coset $W_{P} w_{m i n} W_{Q}$, then we define the following subset of the set $\Delta$ of simple roots of $G$ :

$$
K_{w_{\text {min }}}=\left\{s \in \Delta(P) \mid w_{\text {min }}^{-1} s w_{\text {min }} \in \Delta(Q)\right\},
$$

where for any parabolic subgroup $R \subset G, \Delta(R) \subset \Delta$ is such that the associated reflections, together with $B$, generate $R$. Denote by $R_{w_{\text {min }}}$ the parabolic subgroup of $L$ generated by $K_{w_{m i n}}$ and $B \cap L$. We have the following geometric description of parabolic orbits : 
Theorem 4 (9, Thm. 1.1). Consider the P-orbit $\mathcal{O}$ associated to a double coset $W_{P} w_{\min } W_{Q}$, where $P$ is a parabolic subgroup associated to a cominuscule weight. Then there exists a representation $V_{w_{\text {min }}}$ of $R_{w_{\text {min }}}$ such that $\mathcal{O} \cong L \times_{R_{w_{m i n}}} V_{w_{\text {min }}}$ and the map $\mathcal{O} \rightarrow L / R_{w_{\text {min }}}$ is a vector bundle.

Remark 5. - An analogous result is proved in [10, Prop. 5].

- Note that if $P$ is not associated to a cominuscule weight, we still have a locally trivial map with affine fibers, but it is no longer a vector bundle.

A consequence of Thm. 4 is that the cohomology ring of the parabolic orbit $\mathcal{O}$ and of the generalized flag variety $L / R_{w_{m i n}}$ are isomorphic (see [4, Chap. 3]), which will help us to find decompositions of the Hasse diagrams in Section 4

\subsection{Parabolic orbits associated to cominuscule weights in the classical Grassmannians}

For us, a classical Grassmannian will be a homogeneous space $X=G / Q$, where $G$ is of type $A_{n}$, $B_{n}, C_{n}$ or $D_{n}$ and $Q$ is a maximal parabolic subgroup of $G$. In type $A_{n}$, it corresponds to the usual Grassmannians $\mathrm{G}(m, n+1)$ for $1 \leq m \leq n$, while in type $C_{n}$, we get the symplectic Grassmannians IG $(m, 2 n)$ with $1 \leq m \leq n$. Finally, in type $B_{n}$ (resp. in type $D_{n}$ ), we obtain the odd orthogonal (resp. even orthogonal) Grassmannians $\mathrm{OG}(m, 2 n+1)(\operatorname{resp} . \mathrm{OG}(m, 2 n))$, where $1 \leq m \leq n$. In type $D_{n}$, we furthermore exclude the case where $m=n-1$, since it corresponds to a variety with Picard number two.

We start by giving the list of cominuscule weights, including the exceptional cases :

Table 1: Cominuscule weights

\begin{tabular}{lll}
\hline Type & Classical Grassmannians & Cominuscule weights \\
\hline$A_{n}$ & $\mathrm{G}(m, n+1) 1 \leq m \leq n$ & $\omega_{i}(1 \leq i \leq n)$ \\
$B_{n}$ & $\mathrm{OG}(m, 2 n+1) 1 \leq m \leq n$ & $\omega_{1}$ \\
$C_{n}$ & $\mathrm{IG}(m, 2 n) 1 \leq m \leq n$ & $\omega_{n}$ \\
$D_{n}$ & $\mathrm{OG}(m, 2 n) 1 \leq m \leq n, m \neq n-1$ & $\omega_{1}, \omega_{n-1}, \omega_{n}$ \\
$E_{6}$ & $E_{6} / P_{j} 1 \leq j \leq 6$ & $\omega_{1}, \omega_{6}$ \\
$E_{7}$ & $E_{7} / P_{j} 1 \leq j \leq 7$ & $\omega_{7}$ \\
\hline
\end{tabular}

In the following sections, following Thm. 4, we describe the parabolic orbits associated to the above cominuscule weights for classical Grassmannians. We will not treat the exceptional cases in general since in these examples, flags and Schubert varieties are not so easily described. We will only mention the case of the Cayley plane $E_{6} / P_{1}$ in Section 4 . However, it would probably be possible to get similar results for all exceptional cases, using the description of flags introduced by Iliev and Manivel in [8] for type $E_{6}$ and by Garibaldi in [6] for type $E_{7}$.

We will denote by $P_{\omega_{i}}$ the maximal parabolic subgroup containing the Borel subgroup $B$ and associated to the cominuscule fundamental weight $\omega_{i}$. In 2.2.1. we give a geometric description of the $P_{\omega_{i}}$-orbits, whereas in 2.2 .2 , we give a combinatorial description of the double cosets indexing them.

\subsubsection{Geometric description of parabolic orbits}

First we need to recall the characterization of the flag stabilized by the Borel subgroup $B$ in each of the classical types :

Type $\mathrm{A}_{n}: B$ is the stabilizer of a (uniquely defined) complete flag

$$
0=E_{0} \subset E_{1} \subset \cdots \subset E_{n} \subset E_{n+1}=\mathbb{C}^{n+1},
$$

the element $E_{i}$ being an $i$-dimensional subspace of $\mathbb{C}^{n+1}$. 
Type $\mathrm{B}_{n}: B$ is the stabilizer of a type $B_{n}$ complete isotropic flag

$$
0=E_{0} \subset E_{1} \subset \cdots \subset E_{n} \subset E_{n+1} \subset \cdots \subset E_{2 n} \subset E_{2 n+1}=\mathbb{C}^{2 n+1},
$$

where the vector spaces $E_{1}, \ldots, E_{n}$ are isotropic and for each $1 \leq i \leq n$, we have $E_{n+i}=E_{n+1-i}^{\perp}$.

Type $\mathrm{C}_{n}: B$ is the stabilizer of a type $C_{n}$ complete isotropic flag

$$
0=E_{0} \subset E_{1} \subset \cdots \subset E_{n} \subset E_{n+1} \subset \cdots \subset E_{2 n}=\mathbb{C}^{2 n},
$$

where the $E_{i}$ are isotropic and for each $0 \leq i \leq n$, we have $E_{n+i}=E_{n-i}^{\perp}$.

Type $\mathrm{D}_{n}: B$ is the stabilizer of a type $D_{n}$ complete isotropic flag

$$
0=E_{0} \subset \cdots \subset E_{n-2} \underset{E_{n}^{\prime}}{\subset} \underset{\subset}{E_{n}} \subset E_{n+1} \subset \cdots \subset E_{2 n}=\mathbb{C}^{2 n}
$$

where the vector spaces $E_{1}, \ldots, E_{n-2}$ are isotropic, $E_{n}$ is a type 1 maximal isotropic subspace, $E_{n}^{\prime}$ a type 2 isotropic subspace, $E_{n+1}=\left(E_{n} \cap E_{n}^{\prime}\right)^{\perp}$ and for each $1 \leq i \leq n-1$, $E_{n+1+i}=E_{n-1-i}^{\perp}$.

Now we prove that $P$-orbits associated to cominuscule weights in the classical Grassmannians can be described by the relative position of their elements with respect to a certain partial flag associated to the cominuscule weight defining $P$. In the following proposition, the unique complete flag stabilized by the Borel subgroup will be denoted as above .

Proposition 6. 1. If $X=\mathrm{G}(m, n+1)$ and $P=P_{\omega_{i}}$ for $1 \leq i \leq n$, then the P-orbits are the

$$
\mathcal{O}_{d}:=\left\{\Sigma \in X \mid \operatorname{dim}\left(\Sigma \cap E_{i}\right)=d\right\},
$$

for $\max (0, i+m-n-1) \leq d \leq \min (m, i)$.

2. a) If $X=\mathrm{OG}(m, 2 n+1)$ with $m<n$ and $P=P_{\omega_{1}}$, then the $P$-orbits are

$$
\begin{aligned}
& \mathcal{O}_{0}:=\left\{\Sigma \in X \mid \Sigma \not \subset E_{1}^{\perp}\right\}, \\
& \mathcal{O}_{1}:=\left\{\Sigma \in X \mid \Sigma \subset E_{1}^{\perp} \text { and } \Sigma \not \supset E_{1}\right\}, \\
& \mathcal{O}_{2}:=\left\{\Sigma \in X \mid \Sigma \supset E_{1}\right\} .
\end{aligned}
$$

b) If $X=\mathrm{OG}(n, 2 n+1)$ and $P=P_{\omega_{1}}$, then the $P$-orbits are

$$
\begin{aligned}
& \mathcal{O}_{0}:=\left\{\Sigma \in X \mid \Sigma \not \supset E_{1}\right\}, \\
& \mathcal{O}_{1}:=\left\{\Sigma \in X \mid \Sigma \supset E_{1}\right\} .
\end{aligned}
$$

3. If $X=\mathrm{IG}(m, 2 n)$ and $P=P_{\omega_{n}}$, then the $P$-orbits are the

$$
\mathcal{O}_{d}:=\left\{\Sigma \in X \mid \operatorname{dim}\left(\Sigma \cap E_{n}\right)=d\right\}
$$

for $0 \leq d \leq m$.

4. a) If $X=\mathrm{OG}(m, 2 n)$ with $m<n-1$ and $P=P_{\omega_{1}}$, then the $P$-orbits are defined as in case [2a.

b) If $X=\mathrm{OG}(m, 2 n)$ with $m<n-1$ and $P=P_{\omega_{n-1}}$, then the $P$-orbits are the

$$
\mathcal{O}_{d}:=\left\{\Sigma \in X \mid \operatorname{dim}\left(\Sigma \cap E_{n}^{\prime}\right)=d\right\}
$$

for $0 \leq d \leq m$. 
c) If $X=\mathrm{OG}(m, 2 n)$ with $m<n-1$ and $P=P_{\omega_{n}}$, then the $P$-orbits are defined as in case 4b. with $E_{n}^{\prime}$ replaced by $E_{n}$.

d) If $X=\mathrm{OG}(n, 2 n) \cong \mathrm{OG}(n-1,2 n-1)$ and $P=P_{\omega_{1}}$, then the $P$-orbits are defined as in case $2 b$.

e) If $X=\mathrm{OG}(n, 2 n)$ and $P=P_{\omega_{n-1}}$, then the $P$-orbits are the

$$
\mathcal{O}_{d}:=\left\{\Sigma \in X \mid \operatorname{dim}\left(\Sigma \cap E_{n}^{\prime}\right)=2 d+\epsilon^{\prime}\right\}
$$

for $0 \leq d \leq\left\lfloor\frac{n-1}{2}\right\rfloor$, where $\epsilon^{\prime}=0$ if $n$ is odd and 1 if $n$ is even.

f) If $X=\mathrm{OG}(n, 2 n)$ and $P=P_{\omega_{n}}$, then the $P$-orbits are defined as in case [e, with $E_{n}^{\prime}$ replaced by $E_{n}$ and $\epsilon^{\prime}$ replaced by $\epsilon:=1-\epsilon^{\prime}$.

Proof. The parabolic subgroup $P$ is the stabilizer of the following partial flags :

- $E_{i}$ in case 1 ;

- $E_{1} \subset E_{1}^{\perp}$ in cases $2,4 \mathrm{a}$ and $4 \mathrm{~d}$;

- $E_{n}=E_{n}^{\perp}$ in cases 3 , $4 \mathrm{c}$ and $4 \mathrm{f}$;

- $E_{n}^{\prime}=E_{n}^{\prime \perp}$ in cases $4 \mathrm{~b}$ and $4 \mathrm{e}$.

Hence the dimensions of the intersections with each element of these partial flags are constant on the $P$-orbits, and conversely, the sets where these dimensions are constant are exactly the $P$-orbits.

We conclude the section by giving in each classical type an explicit description of the fibration introduced in Thm. 4. In the following result, the orbits $\mathcal{O}_{d}$ are the ones defined in Prop. 6

Proposition 7. 1. If $X=\mathrm{G}(m, n+1)$ and $P=P_{\omega_{i}}$ for $1 \leq i \leq n$, then the fibrations are the

$$
\begin{array}{ccc}
\mathcal{O}_{d} & \rightarrow & \mathrm{G}\left(d, E_{i}\right) \times G\left(m-d, \mathbb{C}^{n+1} / E_{i}\right) \\
\Sigma & \mapsto & \left(\Sigma \cap E_{i}, \Sigma /\left(\Sigma \cap E_{i}\right)\right)
\end{array}
$$

2. a) If $X=\mathrm{OG}(m, 2 n+1)$ with $m<n$ and $P=P_{\omega_{1}}$, then the fibrations are the

$$
\begin{array}{ccc}
\mathcal{O}_{d} & \rightarrow & \mathrm{OG}\left(m-\epsilon, E_{1}^{\perp} / E_{1}\right) \\
\Sigma & \mapsto & {\left[\Sigma \cap E_{1}^{\perp}\right]}
\end{array}
$$

where $\epsilon=1$ if $d=0,2$ and $\epsilon=0$ if $d=1$.

b) If $X=\mathrm{OG}(n, 2 n+1)$ and $P=P_{\omega_{1}}$, then the fibrations are the

$$
\begin{array}{ccc}
\mathcal{O}_{d} & \rightarrow & \mathrm{OG}\left(n-1, E_{1}^{\perp} / E_{1}\right) \\
\Sigma & \mapsto & {\left[\Sigma \cap E_{1}^{\perp}\right]}
\end{array}
$$

3. If $X=\mathrm{IG}(m, 2 n)$ and $P=P_{\omega_{n}}$, then the fibrations are the

$$
\begin{array}{ccc}
\mathcal{O}_{d} & \rightarrow & \mathrm{F}\left(d, n-m+d ; E_{n}\right) \\
\Sigma & \mapsto & \left(\left(\Sigma \cap E_{n}\right) \subset\left(\Sigma^{\perp} \cap E_{n}\right)\right)
\end{array}
$$

4. a) If $X=\mathrm{OG}(m, 2 n)$ with $m<n-1$ and $P=P_{\omega_{1}}$, then the fibrations are defined as in case 2a.

b) If $X=\mathrm{OG}(m, 2 n)$ with $m<n-1$ and $P=P_{\omega_{n-1}}$, then the fibrations are

$$
\begin{array}{ccc}
\mathcal{O}_{d} & \rightarrow & \mathrm{F}\left(d, n-m+d ; E_{n}^{\prime}\right) \\
\Sigma & \mapsto & \left(\left(\Sigma \cap E_{n}^{\prime}\right) \subset\left(\Sigma^{\perp} \cap E_{n}^{\prime}\right)\right)
\end{array}
$$

c) If $X=\mathrm{OG}(m, 2 n)$ with $m<n-1$ and $P=P_{\omega_{n}}$, then the fibrations are defined as in case 3. 
d) If $X=\mathrm{OG}(n, 2 n) \cong \mathrm{OG}(n-1,2 n-1)$ and $P=P_{\omega_{1}}$, then the fibrations are defined as in case 26 .

e) If $X=\mathrm{OG}(n, 2 n)$ and $P=P_{\omega_{n-1}}$, then the fibrations are

$$
\begin{array}{ccc}
\mathcal{O}_{d} & \rightarrow & \mathrm{G}\left(2 d+\epsilon^{\prime}, E_{n}^{\prime}\right) \\
\Sigma & \mapsto & \Sigma \cap E_{n}^{\prime}
\end{array}
$$

where $\epsilon^{\prime}=0$ if $n$ is odd and 1 if $n$ is even.

f) If $X=\mathrm{OG}(n, 2 n)$ and $P=P_{\omega_{n}}$, then the fibrations are defined as in case $4 e$, with $E_{n}^{\prime}$ replaced by $E_{n}$ and $\epsilon^{\prime}$ replaced by $\epsilon:=1-\epsilon^{\prime}$.

Proof. We only describe Cases 1, 2a, 3 and 4f with $n$ even. The other cases are very similar.

1. Since $\mathcal{O}_{d}=\left\{\Sigma \in X \mid \operatorname{dim}\left(\Sigma \cap E_{i}\right)=d\right\}$, the map is well defined. Moreover, the fiber at a pair $\left(\Sigma_{1}, \Sigma_{2}\right) \in \mathrm{G}\left(d, E_{i}\right) \times G\left(m-d, \mathbb{C}^{n+1} / E_{i}\right)$ is

$$
\left\{\Sigma_{1} \oplus \Sigma^{\prime} \mid \operatorname{dim} \Sigma^{\prime}=m-d, \Sigma^{\prime}=\Sigma_{2} \bmod E_{i}\right\} \cong \mathbb{C}^{\operatorname{dim} \Sigma_{2} \times \operatorname{dim} E_{i}}=\mathbb{C}^{(m-d) i} .
$$

2a For $d=0$, the fiber over $\Sigma_{1} \in \mathrm{OG}\left(m-1, E_{1}^{\perp} / E_{1}\right)$ is

$$
\begin{aligned}
& \left\{\Sigma^{\prime} \oplus L \mid \Sigma^{\prime}=\Sigma_{1} \bmod E_{1}, L \subset \Sigma_{1}^{\perp} \backslash E_{1}^{\perp}, L \text { isotropic }\right\} \\
\cong & \mathbb{C}^{\operatorname{dim} \Sigma_{1} \times \operatorname{dim} E_{1}} \times \mathbb{C}^{\operatorname{dim} \Sigma_{1}^{\perp}-\operatorname{dim} \Sigma_{1}-\operatorname{dim} L-1}=\mathbb{C}^{2 n-m} .
\end{aligned}
$$

For $d=1$, the fiber over $\Sigma_{1} \in \mathrm{OG}\left(m, E_{1}^{\perp} / E_{1}\right)$ is

$$
\left\{\Sigma^{\prime} \mid \Sigma^{\prime}=\Sigma_{1} \bmod E_{1}\right\} \cong \mathbb{C}^{\operatorname{dim} E_{1} \operatorname{dim} \Sigma_{1}}=\mathbb{C}^{m} .
$$

Finally, for $d=2$, the map is an isomorphism.

3. The fiber over $\left(\Sigma_{1} \subset \Sigma_{2}\right) \in \mathrm{F}\left(d, n-m+d ; E_{n}\right)$ is

$$
\begin{aligned}
& \left\{\Sigma_{1} \oplus \Sigma^{\prime} \mid \operatorname{dim} \Sigma^{\prime}=m-d, \Sigma^{\prime}=\Sigma_{2}^{\perp} \bmod E_{n}, \Sigma^{\prime} \subset \Sigma_{1}^{\perp} \text { isotropic }\right\} \\
\cong & \mathbb{C}^{\operatorname{dim} \Sigma^{\prime}\left(\operatorname{dim} E_{n}-\operatorname{dim} \Sigma_{1}\right)-\frac{\operatorname{dim} \Sigma^{\prime}\left(\operatorname{dim} \Sigma^{\prime}-1\right)}{2}}=\mathbb{C}^{(m-d)(n-d)-\frac{(m-d)(m-d-1)}{2}} .
\end{aligned}
$$

4f) We assume $n$ is even. The fiber over $\Sigma_{1} \in \mathrm{G}\left(2 d, E_{n}\right)$ is

$$
\begin{aligned}
& \left\{\Sigma_{1} \oplus \Sigma^{\prime} \mid \operatorname{dim} \Sigma^{\prime}=m-2 d, \Sigma^{\prime}=\Sigma_{1}^{\perp} \bmod E_{n} \Sigma^{\prime} \subset \Sigma_{1}^{\perp}, \Sigma^{\prime} \text { isotropic }\right\} \\
\cong & \mathbb{C}^{\operatorname{dim} \Sigma^{\prime 2}-\frac{\operatorname{dim} \Sigma^{\prime}\left(\operatorname{dim} \Sigma^{\prime}-1\right)}{2}}=\mathbb{C}^{(n-2 d)^{2}-\frac{(n-2 d)(n-2 d-1)}{2}} .
\end{aligned}
$$

Remark 8. In Thm. 4, the fibrations for parabolic orbits are described combinatorially. Tedious but straightforward calculations show that these fibrations are indeed the same as those described in the above proposition.

\subsubsection{Combinatorial description of parabolic orbits}

We begin by recalling the description of the elements of the Weyl group in type $A_{n}$ (respectively in types $B_{n}, C_{n}$ and $D_{n}$ ) as permutations (resp. signed permutations) of $\{1, \ldots, n\}$. We do not have such a description in the exceptional cases.

In type $A$, the Weyl group is $W=\mathfrak{S}_{n}$, and we denote $w \in W$ as $w=\left(a_{1}, \ldots, a_{n}\right)$ where $\{1, \ldots, n\}=\left\{a_{1}, \ldots, a_{n}\right\}$, which means that $w(i)=a_{i}$.

In types $B_{n}$ and $C_{n}$, the Weyl group is $W=\mathfrak{S}_{n} \ltimes \mathbb{Z}_{2}^{n}$, and we denote $w \in W$ as $w=\left(b_{1}, \ldots, b_{n}\right)$, where $b_{i}=a_{i}$ or $-a_{i}$ and $\{1, \ldots, n\}=\left\{a_{1}, \ldots, a_{n}\right\}$, which means that $w(i)=a_{i}$ if $b_{i}=a_{i}$ and $w(i)=a_{i}$ if $b_{i}=-a_{i}$.

Finally, in type $D_{n}$, the Weyl group is $W=\mathfrak{S}_{n} \ltimes \mathbb{Z}_{2}^{n-1}$, and we denote elements of $W$ as in the previous case, with the additional condition that the number of negative parts $-a_{i}$ should be even.

We can now state a proposition describing, for all the classical types, the double coset $\mathcal{E}_{d} \in$ $W_{P} \backslash W / W_{Q}$ indexing the $P$-orbit $\mathcal{O}_{d}$ defined in Prop. 6: 
Proposition 9. 1. If $X=\mathrm{G}(m, n+1)$ and $P=P_{\omega_{i}}$ for $1 \leq i \leq n$, then

$$
\mathcal{E}_{d}=\{w \in W \mid \#\{1 \leq j \leq m \mid w(j) \leq i\}=d\} .
$$

2. a) If $X=\mathrm{OG}(m, 2 n+1)$ with $m<n$ and $P=P_{\omega_{1}}$, then

$$
\begin{aligned}
& \mathcal{E}_{0}=\{w \in W \mid \exists 1 \leq j \leq m, w(j)=-1\} \\
& \mathcal{E}_{1}=\{w \in W \mid \nexists 1 \leq j \leq m, w(j) \in\{1,-1\}\} \\
& \mathcal{E}_{2}=\{w \in W \mid \exists 1 \leq j \leq m, w(j)=1\} .
\end{aligned}
$$

b) If $X=\mathrm{OG}(n, 2 n+1)$ and $P=P_{\omega_{1}}$, then

$$
\begin{aligned}
& \mathcal{E}_{0}=\{w \in W \mid \exists 1 \leq j \leq n, w(j)=-1\} \\
& \mathcal{E}_{1}=\{w \in W \mid \exists 1 \leq j \leq n, w(j)=1\} .
\end{aligned}
$$

3. If $X=\mathrm{IG}(m, 2 n)$ and $P=P_{\omega_{n}}$, then

$$
\mathcal{E}_{d}=\{w \in W \mid \#\{1 \leq j \leq m \mid w(j)>0\}=d\} .
$$

4. a) If $X=\mathrm{OG}(m, 2 n)$ with $m<n-1$ and $P=P_{\omega_{1}}$, then $\mathcal{E}_{d}$ is defined as in case 2a.

b) If $X=\mathrm{OG}(m, 2 n)$ with $m<n-1$ and $P=P_{\omega_{n-1}}$, then

$$
\begin{aligned}
\mathcal{E}_{d}= & \{w \in W \mid \#\{j \leq m \mid w(j)>0\}=d, w(j) \neq n,-n \forall j \leq m\} \\
& \cup\{w \mid \#\{j \leq m \mid w(j)>0\}=d-1, \exists j \leq m, w(j)=-n\} \\
& \cup\{w \mid \#\{j \leq m \mid w(j)>0\}=d+1, \exists j \leq m, w(j)=n\} .
\end{aligned}
$$

c) If $X=\mathrm{OG}(m, 2 n)$ with $m<n-1$ and $P=P_{\omega_{n}}$, then $\mathcal{E}_{d}$ is defined as in case 3

d) If $X=\mathrm{OG}(n, 2 n) \cong \mathrm{OG}(n-1,2 n-1)$ and $P=P_{\omega_{1}}$, then $\mathcal{E}_{d}$ is defined as in case 2b.

e) If $X=\mathrm{OG}(n, 2 n)$ and $P=P_{\omega_{n-1}}$, then

$$
\begin{aligned}
\mathcal{E}_{d}= & \left\{w \in W \mid \#\{j \mid w(j)>0\}=2 d+\epsilon^{\prime}-1 \text { and } \exists j, w(j)=-n\right\} \\
& \cup\left\{w \in W \mid \#\{w(j)>0\}=2 d+\epsilon^{\prime}+1 \text { and } \exists j, w(j)=n\right\},
\end{aligned}
$$

where $\epsilon^{\prime}=0$ if $n$ is odd and 1 if $n$ is even.

f) If $X=\mathrm{OG}(n, 2 n)$ and $P=P_{\omega_{n}}$, then

$$
\mathcal{E}_{d}=\{w \in W \mid \#\{j \mid w(j)>0\}=2 d+\epsilon\},
$$

where $\epsilon=1-\epsilon^{\prime}$.

Proof. The arguments for each case being similar, we only prove the proposition in Case $4 \mathrm{~b}$, which is a little more complicated than the others.

Here the Weyl groups are $W=\mathfrak{S}_{n} \ltimes \mathbb{Z}_{2}^{n-1}, W_{P}=\mathfrak{S}_{n}$ and $W_{Q}=\mathfrak{S}_{m} \times\left(\mathfrak{S}_{n-m} \ltimes \mathbb{Z}_{2}^{n-m-1}\right)$. As in the beginning of the section, we will denote elements of $W$ as signed permutations $w=\left(b_{1}, \ldots, b_{n}\right)$.

The action of $W_{Q}$ on the right permutes the $m$ first entries $b_{1}, \ldots, b_{m}$ of $w$ on one hand, and the $n-m$ last entries $b_{m+1}, \ldots, b_{n}$ on the other hand, and changes the sign of these last entries while keeping the total number of minus signs even. Hence the minimal length representatives of classes in $W / W_{Q}$ are of the form :

$$
w=\left(u_{1}<\cdots<u_{l},-z_{m-l}<\cdots<-z_{1}, v_{1}<\cdots<v_{n-m-1},(-1)^{m-l} v_{n-m}\right),
$$

where $0 \leq l \leq m,\left\{u_{i}\right\} \cup\left\{z_{r}\right\} \cup\left\{v_{j}\right\}=\{1, \ldots, n\}$ and $v_{n-m-1}<v_{n-m}$.

Moreover, the action of $W_{P}$ on the right permutes the $n-1$ values $1, \ldots, n-1$ and exchanges $n-1$ and $n$ while changing their signs. Hence the minimal length representatives of double cosets in $W_{P} \backslash W / W_{Q}$ are of the form :

$$
w_{0}=i d \text { or } w_{d}=(1<\cdots<d-1<n,-n+1<\cdots<-n+m-d, \underline{v}),
$$


where $1 \leq d \leq m$ and

$$
\underline{v}=\left(d<\cdots<n-m+d-2,(-1)^{m-d}(n-m+d-1)\right) .
$$

Now it is enough to prove that all elements of the set $\mathcal{E}_{d}$ defined in the statement of the proposition are in the same double coset as $w_{d}$.

First suppose $w \in W$ is such that $\#\{j \leq m \mid w(j)<0\}=d$ and $w(j) \neq n,-n$ for all $j \leq m$. Using the action of $W_{Q}$ on the right, we see that $w$ is in the same double coset as

$$
w^{1}=\left(a_{1}<\cdots<a_{d},-b_{m-d}<\cdots<-b_{1}, c_{1}<\cdots<c_{n-m-1},(-1)^{m-d} n\right) .
$$

Using (several times) the action of the simple reflections $s_{1}, \ldots, s_{n-1}$ of $W_{P}$ on the left (which together permute the values from 1 to $n-1$ ), we deduce that $w^{1}$ is in the same double coset as

$$
w^{2}=(1<\cdots<d,-n+1<\cdots<-n+m-d, \underline{v}),
$$

where $\underline{v}=\left(d+1<\cdots<n-m+d-1,(-1)^{m-d} n\right)$. Then applying the simple reflection $s_{n} \in W_{P}$ on the left, we get

$$
w^{3}=(1<\cdots<d<n,-n+2<\cdots<-n+m-d, \underline{v}),
$$

where $\underline{v}=\left(d+1<\cdots<n-m+d-1,(-1)^{m-d+1}(n-1)\right)$.

Finally, using the action of the simple reflections $s_{1}, \ldots, s_{n-1}$ of $W_{P}$ on the left, we obtain the element $w^{4}=w_{d}$, which proves that $w$ is in the same double coset as $w_{d}$.

The reasoning in the two other situations ( $\#\{j \leq m \mid w(j)>0\}=d-1$ and $\exists j \leq m, w(j)=-n$ on one hand, $\#\{j \leq m \mid w(j)>0\}=d+1$ and $\exists j \leq m, w(j)=n$ on the other hand) being very similar, this concludes the proof.

Notation 10. Let $w \in W$ be an element of the Weyl group. Then $w$ belongs to one of the double cosets $\mathcal{E}_{d}$ defined in the statement of the proposition, and we define the integer $d(w):=d$.

\section{$3 \quad$ Link between $P$-orbits and the quantum product}

Here we describe the link between Thm. 1 and parabolic orbits for homogeneous spaces $X=G / Q$, where $Q$ is a maximal parabolic subgroup. Since $Q$ is maximal, we have $\Phi^{\vee} / \Phi_{Q}^{\vee} \cong \mathbb{Z}$. Hence for each $w \in W$, we may define an integer

$$
\delta(w):=\eta_{Q}\left(\omega_{i}^{\vee}-w^{-1}\left(\omega_{i}^{\vee}\right)\right) .
$$

In the following sections, we will prove that the loci where $\delta(w)$ is constant correspond to the double cosets $\mathcal{E}$ indexing $P$-orbits. For classical Grassmannians, this proves that for every $w \in W^{Q}, \delta(w)$ equals the integer $d(w)$ introduced in Notation 10 .

\subsection{The integer $\delta(w)$ is constant on parabolic orbits.}

We start by proving that $\delta$ is constant on the double cosets $\mathcal{E}=W_{P} w W_{Q}$. Consider $w^{\prime} \in \mathcal{E}$. From the definition of $\mathcal{E}$, it follows that $w^{\prime}$ can be written as $w_{P} w w_{Q}$ for some $w_{P} \in W_{P}$ and $w_{Q} \in W_{Q}$. Denote by $\omega_{i}$ the cominuscule weight defining $P$. Reflections associated to the simple roots will be denoted by $s_{l}$ for $1 \leq l \leq n$.

If $l \neq i$, we have

$$
s_{l}\left(\omega_{i}^{\vee}\right)=\omega_{i}^{\vee}-\left(\alpha_{l}, \omega_{i}^{\vee}\right) \alpha_{l}^{\vee}=\omega_{i}^{\vee},
$$

hence

$$
w_{P}^{-1}\left(\omega_{i}^{\vee}\right)=\omega_{i}^{\vee}
$$

Now consider $e:=\eta_{Q}\left(w^{-1}\left(\omega_{i}^{\vee}\right)\right)$. Then by definition of $\eta_{Q}$,

$$
w^{-1}\left(\omega_{i}^{\vee}\right)=e \alpha_{m}^{\vee}+\sum_{p \neq m} c_{p} \alpha_{p}^{\vee},
$$


where the $c_{p}$ are some coefficients. But if $l \neq m$, we have

$$
s_{l}\left(\alpha_{m}^{\vee}\right)=\alpha_{j}^{\vee}-\left(\alpha_{l}, \alpha_{m}^{\vee}\right) \alpha_{l}^{\vee} .
$$

Similarly, for $p \neq m$ and $l \neq p, m$ :

$$
s_{l}\left(\alpha_{p}^{\vee}\right)=\alpha_{p}^{\vee}-\left(\alpha_{l}, \alpha_{p}^{\vee}\right) \alpha_{l}^{\vee},
$$

and if $p \neq m$ and $l=p$ :

$$
s_{p}\left(\alpha_{p}^{\vee}\right)=-\alpha_{p}^{\vee} .
$$

Hence if we apply the reflection $s_{l}$ for $l \neq m$, the coefficient of $\alpha_{m}^{\vee}$ does not change. We conclude that $\eta_{Q}\left(w_{Q}^{-1} w^{-1} \omega_{i}^{\vee}\right)=\eta_{Q}\left(w^{-1} \omega_{i}^{\vee}\right)$. Using Equation (1), we obtain

$$
\eta_{Q}\left(w_{Q}^{-1} w^{-1} w_{P}^{-1} \omega_{i}^{\vee}\right)=\eta_{Q}\left(w^{-1} \omega_{i}^{\vee}\right)
$$

\subsection{The integer $\delta(w)$ changes on different parabolic orbits.}

It is enough to prove that if $w^{\prime} \in \mathcal{E}^{\prime} \cap W^{Q}$ is a successor of $w \in \mathcal{E} \cap W^{Q}$ for the Bruhat order in $W^{Q}$, where $\mathcal{E}$ and $\mathcal{E}^{\prime}$ are two different $P$-orbits, then $\delta\left(w^{\prime}\right)>\delta(w)$.

Since $w$ and $w$ and $w^{\prime}$ do not belong to the same $P$-orbit, we know that $w^{\prime}=s_{\alpha_{0}} w$ for some positive root $\alpha_{0} \in \Phi^{+} \backslash\left(\Phi_{P}^{+} \cap \Phi_{Q}^{+}\right\}$. Indeed, if $\alpha \in \Phi_{P}^{+}$, then the reflection $s_{\alpha}$ is in $W_{P}$, hence stabilizes $\mathcal{E}$ and if $\alpha \in \Phi_{Q}^{+}$, then $w^{\prime}=w$ in $W / W_{Q}$. Moreover, we have $l_{Q}\left(w^{\prime}\right)=l_{Q}(w)+1$, where $l_{Q}$ is the length function of $W^{Q}$.

We set $L_{Q}(w):=\left\{\alpha \in \Phi^{+} \backslash \Phi_{Q}^{+} \mid w(\alpha) \in \Phi^{-}\right\}$. There exists $\beta_{0} \in \Phi^{+} \backslash \Phi_{Q}^{+}$such that $w\left(\beta_{0}\right)=$ $\alpha_{0}$. Indeed, if it were not the case, then for all $\alpha \in L_{Q}\left(w^{\prime}\right)$, we would have $s_{\alpha_{0}} w(\alpha) \in \Phi^{-}$and $w(\alpha) \neq \alpha_{0}$, hence $w(\alpha) \in \Phi^{-}$and $\alpha \in L_{Q}(w)$. This would mean that $l_{Q}\left(w^{\prime}\right) \leq l_{Q}(w)$, which is absurd.

Let us now compute $\delta\left(w^{\prime}\right)$ :

$$
\delta\left(w^{\prime}\right)=\eta_{Q}\left(\omega_{i}^{\vee}-w^{-1} s_{\alpha_{0}}\left(\omega_{i}^{\vee}\right)\right)=\delta(w)+\left(\alpha_{0}, \omega_{i}^{\vee}\right) \eta_{Q}\left(w^{-1} \alpha_{0}^{\vee}\right) .
$$

Since $\alpha_{0} \in \Phi^{+} \backslash \Phi_{P}^{+}$, we have $\left(\alpha_{0}, \omega_{i}^{\vee}\right)>0$. Moreover, $w\left(\beta_{0}\right)=\alpha_{0}$ implies that $w^{-1}\left(\alpha_{0}^{\vee}\right)=\beta_{0}^{\vee}$, and $\eta_{Q}\left(\beta_{0}\right)>0$ since $\beta_{0} \in \Phi^{+} \backslash \Phi_{Q}^{+}$. Finally $\delta\left(w^{\prime}\right)>\delta(w)$ as required.

We conclude that the loci

$$
\left\{w \in W^{Q} \mid \delta(w)=d\right\}
$$

coincide with the sets $\mathcal{E} \cap W^{Q}$.

\section{Decomposition of the Hasse diagram}

In 2, Chaput, Manivel and Perrin relate the quantum product by the point class in minuscule varieties with a decomposition of their Hasse diagram. The Hasse diagram $\mathcal{H}$ of a homogeneous space with Picard rank one is the diagram of the multiplication by the hyperplane class $h$. More precisely, its vertices are the Schubert classes $\sigma_{w}$ for $w \in W^{Q}$ and $\sigma_{v}$ and $\sigma_{w}$ are related by an arrow of multiplicity $r$ if and only if $\sigma_{w}$ appears with multiplicity $r$ in the cup-product $\sigma_{v} \cup h$.

The results of previous sections enable us to find decompositions of the Hasse diagram in the non-minuscule case, corresponding to the quantum product by the Schubert classes $\sigma_{v_{i}}$ associated to cominuscule weights introduced in the statement of $\mathrm{Thm}$. 1 .

Let $\mathcal{O}$ be a $P$-orbit of $X$. It is the union of the Schubert cells $C_{w} \subset X$ for all $w$ in the associated double coset $\mathcal{E}$. The set $\mathcal{E} \cap W^{Q}$ being an interval (cf Prop. 3), we denote it as $\mathcal{E} \cap W^{Q}=\left[w_{\min }, w_{\max }\right]$. From Thm. 1. we know that $\mathcal{O}$ is a vector bundle over the generalized flag variety $F:=L / R_{w_{\text {min }}}$.

Here we state a result relating the Hasse diagrams of the parabolic orbit $\mathcal{O}$ with a similar diagram for the flag variety $F$ : 
Theorem 11. Let $\psi: \mathcal{O} \rightarrow F$ be the fibration, $i: \mathcal{O} \hookrightarrow X$ the natural embedding and $h$ the hyperplane class of $X$. Then :

1. There exists a class $h^{\prime} \in \mathrm{H}^{2}(F)$ such that $i^{*} h=\psi^{*} h^{\prime}$;

2. The Hasse diagram of $\mathcal{O}$ is isomorphic to the diagram of the multiplication by $h^{\prime}$ in $F$.

Proof. 1. Since $i^{*} h \in \mathrm{H}^{2}(\mathcal{O}) \cong \mathrm{H}^{2}(F)$, there exists a (unique) $h^{\prime} \in \mathrm{H}^{2}(F)$ such that $i^{*} h=\psi^{*} h^{\prime}$.

2. There exists an isomorphism $W^{F} \cong \mathcal{E} \cap W^{Q}$, where $W^{F}$ is the set of minimal length representatives of $W_{L} / W_{R_{w_{\text {min }}}}$. Indeed, let $C_{u}^{F}$ be a Schubert cell of $F$. Since $\psi$ is a vector bundle, its inverse image $\psi^{-1}\left(C_{u}^{F}\right)$ is a Schubert cell of $X$, which we denote by $C_{\phi(u)}^{X}$, where $\phi(u) \in W^{Q}$. Since $C_{\phi(u)}^{X} \subset \mathcal{O}$, we have $\phi(u) \in \mathcal{E} \cap W^{Q}$, and $\phi$ is the desired isomorphism. It yields a correspondence between the vertices of the Hasse diagram of $\mathcal{O}$ and those of the diagram of the multiplication by the class $h^{\prime}$ in $F$.

Now we study the correspondence between the edges of both diagrams. Assume that

$$
\left[Y_{w}\right] \cup h^{\prime}=\sum_{v} a_{v}\left[Y_{v}\right]
$$

where $Y_{v}$ denotes the Schubert variety of $F$ associated to the element $v$. This means that a generic hyperplane section of $Y_{w}$ is rationally equivalent to the union of the $Y_{v}$ with multiplicities $a_{v}$. Let $Y_{u}$ be a Schubert variety of $F$. Its inverse image $\psi^{-1}\left(Y_{u}\right)$ is the closure in $\mathcal{O}$ of the Schubert cell $C_{\phi(u)}^{X}$, hence it is the intersection of $\mathcal{O}$ with the Schubert variety $X_{\phi(u)}$. Thus $X_{\phi(w)} \cap \mathcal{O}$ is rationally equivalent to the union of the $X_{\phi(v)} \cap \mathcal{O}$ with multiplicities $a_{v}$. As a consequence, if $H$ is a generic hyperplane, a section $X_{\phi(w)} \cap \mathcal{O} \cap H$ is rationally equivalent to the union of the $X_{\phi(v)} \cap \mathcal{O} \cap H$ with multiplicities $a_{v}$. If we consider the closure in $\overline{\mathcal{O}}$, we deduce that $X_{\phi(w)} \cap H$ is rationally equivalent to the sum of the $X_{\phi(v)}$ with multiplicities $a_{v}$, plus a class $Z$ supported in the boundary $\overline{\mathcal{O}} \backslash \mathcal{O}$. But such a class is rationally equivalent to the union of some Schubert varieties $X_{u}$ contained in $\overline{\mathcal{O}} \backslash \mathcal{O}$, with some multiplicities $b_{u}$. This rational equivalence stays true in the whole of $X=G / P_{J}$. Taking cohomology classes, it means that

$$
\sigma_{\phi(w)} \cup h=\sum_{v} a_{v} \sigma_{\phi(v)}+\sum_{u} b_{u} \sigma_{u}
$$

Since the Schubert varieties $X_{u}$ are contained in $\overline{\mathcal{O}} \backslash \mathcal{O}$, the elements $u \in W^{Q}$ are not contained $\mathcal{E} \cap W^{Q}$. Hence they do not contribute to the arrows of the Hasse diagram of $\mathcal{O}$. This proves that the Hasse diagram of $\mathcal{O}$ has the same arrows as the diagram of the multiplication by the class $h^{\prime}$ in $F$.

We may now conclude by combining the previous results to describe the Hasse diagrams of the classical Grassmannians :

Theorem 12. 1. In types $A_{n}, C_{n}, D_{n}$, and in type $B_{n}$ for odd orthogonal Grassmannians $\mathrm{OG}(m, 2 n+1)$ with $m \neq n-1$, if $\mathcal{O}$ is a parabolic orbit associated to a cominuscule weight $\omega_{i}$, the Hasse diagrams $\mathcal{H}_{\mathcal{O}}$ and $\mathcal{H}_{F}$ of $\mathcal{O}$ and the corresponding flag variety $F$ described in Prop. [6] are isomorphic.

2. In type $B_{n}$ for the odd orthogonal Grassmannian $\mathrm{OG}(n-1,2 n+1)$, if we denote by $\mathcal{O}_{0}, \mathcal{O}_{1}$ and $\mathcal{O}_{2}$ the parabolic orbits associated to the weight $\omega_{1}$ and $F_{0}, F_{1}$ and $F_{2}$ the corresponding flag varieties, we have $\mathcal{H}_{\mathcal{O}_{0}} \cong \mathcal{H}_{F_{0}}$ and $\mathcal{H}_{\mathcal{O}_{2}} \cong \mathcal{H}_{F_{2}}$, but $\mathcal{H}_{\mathcal{O}_{1}}$ corresponds to $\mathcal{H}_{F_{1}}$ with the multiplicities of the arrows doubled.

Proof. In both cases, we apply Thm. 11 to the map $\psi: \mathcal{O} \rightarrow F$ from Prop. 7 and the natural embedding $i: \mathcal{O} \rightarrow X=G / Q$, denoting by $h$ the hyperplane class of $X$. It follows that there exists a unique $h^{\prime} \in \mathrm{H}^{2}(F)$ such that $i^{*} h=\psi^{*}\left(h^{\prime}\right)$, and that the Hasse diagram of $\mathcal{O}$ is isomorphic to the diagram of the multiplication by $h^{\prime}$ in $F$. So to prove Thm. 12 , we only need to prove that $h^{\prime}$ is equal to the hyperplane class of $F$ in types $A_{n}, C_{n}, D_{n}$, and in type $B_{n}$ for odd orthogonal Grassmannians $\mathrm{OG}(m, 2 n+1)$ with $m \neq n-1$, and that it is equal to twice the hyperplane class of 
$F$ otherwise. In 'hyperplane class of $F$ ', we refer of course to the minimal embedding of $F$ inside projective space.

In type $A_{n}$, denote by $\mathcal{S}$ the tautological bundle on $X$ and $\mathcal{S}_{1}, \mathcal{S}_{2}$ the tautological bundles on the product of Grassmannians $F$. By definition $h=c_{1}(\operatorname{det} \mathcal{S})$. Moreover

$$
0 \rightarrow \psi^{*} \mathcal{S}_{1} \rightarrow i^{*} \mathcal{S} \rightarrow \psi^{*} \mathcal{S}_{2} \rightarrow 0
$$

hence $\psi^{*} c_{1}\left(\operatorname{det} \mathcal{S}_{1} \otimes \operatorname{det} \mathcal{S}_{2}\right)=i^{*} h$. It follows that $h^{\prime}=c_{1}\left(\operatorname{det} \mathcal{S}_{1} \otimes \operatorname{det} \mathcal{S}_{2}\right)$, which is indeed the hyperplane class of $F$.

In type $B_{n}$ for $X=\mathrm{OG}(m, 2 n+1)$ with $m<n$, we will prove for each of the three $P$-orbits $\mathcal{O}_{d}$ for $d=0,1,2$ that $i^{*}(\operatorname{det} \mathcal{S})=\psi^{*}\left(\operatorname{det} \mathcal{S}_{1}\right)$, where $\mathcal{S}_{1}$ is the tautological bundle on $F$. Since $c_{1}(\operatorname{det} \mathcal{S})=h$, it will follow that $h^{\prime}=c_{1}\left(\operatorname{det} \mathcal{S}_{1}\right)$. For $d=0$, we have the exact sequences

$$
\begin{aligned}
& 0 \rightarrow \Sigma \cap E_{1}^{\perp} \rightarrow \Sigma \rightarrow \Sigma /\left(\Sigma \cap E_{1}^{\perp}\right) \rightarrow 0 \\
& 0 \rightarrow \Sigma^{\prime} \rightarrow E_{1}^{\perp} / E_{1} \rightarrow E_{1}^{\perp} /\left(\Sigma \cap E_{1}^{\perp} \oplus E_{1}\right) \rightarrow 0 \\
& 0 \rightarrow \Sigma \cap E_{1}^{\perp} \rightarrow \Sigma \cap E_{1}^{\perp} \oplus E_{1} \rightarrow\left(\Sigma \cap E_{1}^{\perp} \oplus E_{1}\right) /\left(\Sigma \cap E_{1}^{\perp}\right) \rightarrow 0,
\end{aligned}
$$

which give the following equalities of determinant bundles

$$
\begin{aligned}
\operatorname{det}(\Sigma) & =\operatorname{det}\left(\Sigma \cap E_{1}^{\perp}\right) \otimes \operatorname{det}\left(\Sigma /\left(\Sigma \cap E_{1}^{\perp}\right)\right) \\
\operatorname{det}\left(\Sigma^{\prime}\right) & =\operatorname{det}\left(\Sigma \cap E_{1}^{\perp} \oplus E_{1}\right) \\
\operatorname{det}\left(\Sigma \cap E_{1}^{\perp} \oplus E_{1}\right) & =\operatorname{det}\left(\Sigma \cap E_{1}^{\perp}\right) \otimes \operatorname{det}\left(\left(\Sigma \cap E_{1}^{\perp} \oplus E_{1}\right) /\left(\Sigma \cap E_{1}^{\perp}\right)\right) .
\end{aligned}
$$

We conclude by using the fact that the quadratic form induces a duality

$$
\Sigma /\left(\Sigma \cap E_{1}^{\perp}\right) \times\left(\Sigma \cap E_{1}^{\perp} \oplus E_{1}\right) /\left(\Sigma \cap E_{1}^{\perp}\right) \rightarrow \mathbb{C} .
$$

For $d=1$, we use the same method, only replacing $\Sigma \cap E_{1}^{\perp}$ with $\Sigma$, and for $d=2$, the result follows from the exact sequence

$$
0 \rightarrow E_{1} \rightarrow \Sigma \rightarrow \Sigma / E_{1} \rightarrow 0 .
$$

Now we have proved that $h^{\prime}=c_{1}\left(\operatorname{det} \mathcal{S}_{1}\right)$, it remains to relate it to the hyperplane class $H$ of $F$. There are two cases :

$$
c_{1}\left(\operatorname{det} \mathcal{S}_{1}\right)= \begin{cases}H & \text { if } m<n-1 \\ 2 H & \text { if } m=n-1\end{cases}
$$

Indeed, $\mathrm{OG}(n-1,2 n-1)$ is projectively isomorphic to $\mathrm{OG}(n-1,2 n-2)$, which is embedded in $\mathbb{P}\left(V_{\omega_{n-1}}\right)$, where $V_{\omega_{n-1}}$ is the half-spin representation. Hence the hyperplane class $H$ is equal to the first Chern class of the line bundle associated to the weight $\omega_{n-1}$, while $\operatorname{det} \mathcal{S}_{1}$ is the line bundle associated to the weight $2 \omega_{n-1}$.

In type $B_{n}$ for $X=\mathrm{OG}(n, 2 n+1)$, we prove as in the non-maximal case that $h^{\prime}=c_{1}\left(\operatorname{det} \mathcal{S}_{1}\right)$ is the hyperplane class of $F$.

In type $C_{n}$, denote by $\mathcal{S}$ the tautological bundle on $X$ and $\mathcal{S}_{1}, \mathcal{S}_{2}$ the tautological bundles on $F$. Since $h=c_{1}(\operatorname{det} \mathcal{S})$ and

$$
\begin{gathered}
0 \rightarrow \Sigma \cap E_{n} \rightarrow \Sigma \rightarrow \Sigma /\left(\Sigma \cap E_{n}\right) \rightarrow 0 \\
0 \rightarrow \Sigma^{\perp} \cap E_{n} \rightarrow E_{n} \rightarrow E_{n} /\left(\Sigma^{\perp} \cap E_{n}\right) \rightarrow 0,
\end{gathered}
$$

we have $h^{\prime}=c_{1}\left(\operatorname{det} \mathcal{S}_{1} \otimes \operatorname{det} \mathcal{S}_{2}\right)$, which is indeed the hyperplane class of $F$.

In type $D_{n}$ for $X=\mathrm{OG}(m, 2 n)$ with $m<n$ or for $X=\mathrm{OG}(n, 2 n)$ with $P=P_{\omega_{1}}$, the result is proven in an analogous way as in types $B_{n}$ and $C_{n}$. This leaves us with the case where $X=\mathrm{OG}(n, 2 n)$ and $P=P_{\omega_{n}}$ or $P_{\omega_{n-1}}$. Here we treat the case $P=P_{\omega_{n}}$, the other being very similar. We use the two exact sequences

$$
\begin{gathered}
0 \rightarrow \Sigma \cap E_{n} \rightarrow \Sigma \rightarrow \Sigma /\left(\Sigma \cap E_{n}\right) \rightarrow 0 \\
0 \rightarrow \Sigma \cap E_{n} \rightarrow E_{n} \rightarrow E_{n} /\left(\Sigma \cap E_{n}\right) \rightarrow 0
\end{gathered}
$$

and the duality $\Sigma /\left(\Sigma \cap E_{n}\right) \times E_{n} /\left(\Sigma \cap E_{n}\right) \rightarrow \mathbb{C}$ to prove that $h^{\prime}=c_{1}\left(\operatorname{det} \mathcal{S}_{1}\right)$. 
Figure 1: $P_{\omega_{4}}$-orbits in $\operatorname{IG}(2,8)$

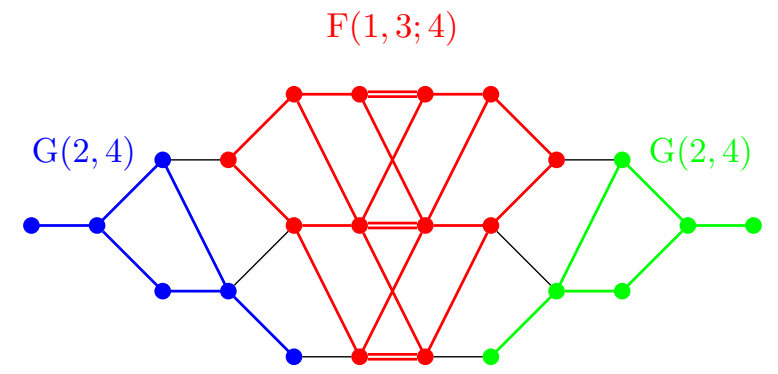

Figure 2: $P_{\omega_{1}}$-orbits in $\mathrm{OG}(3,9)$

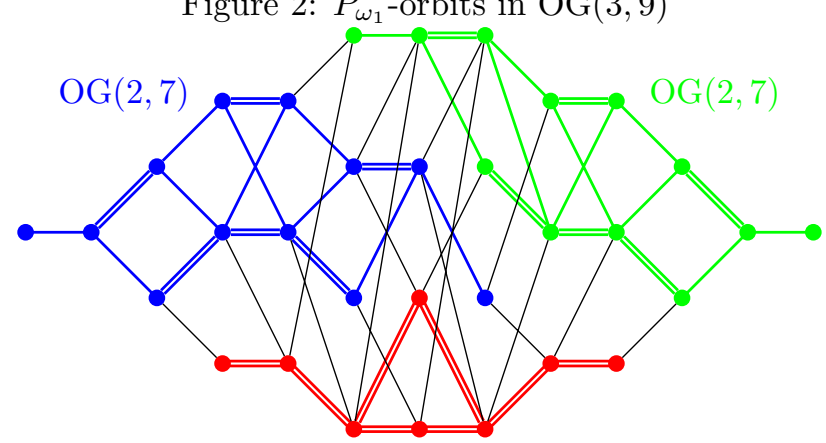

$\mathrm{OG}(3,7)$

Finally, we give some pictures illustrating Thm. 12, We start with a type $C_{n}$ example : the symplectic Grassmannian IG $(2,8)$ in Figure 1. There are three orbits, two being vector bundles over the Grassmannian $\mathrm{G}(2,4)$ and another over the two-step flag variety $\mathrm{F}(1,3 ; 4)$.

Then we consider a type $B_{n}$ example : the odd orthogonal Grassmannian OG(3,9) in Figure 2 There are again three orbits. The first and last are vector bundles over $\mathrm{OG}(2,7)$. For the middle orbit, which is a vector bundle over $\mathrm{OG}(3,7)$, we see as expected that the multiplicity of all arrows is multiplied by 2 .

Finally, let us recall an exceptional example, computed in [2] : the Cayley plane $X=E_{6} / P_{\omega_{1}}=$ $\mathbb{O} \mathbb{P}^{2}$ (see Figure 3). There are three $P_{\omega_{1}}$ orbits. Indeed, we know that a partial $E_{6}$-flag associated to $P_{\omega_{1}}$ simply consists in a point $p_{0} \in X$. The $P_{\omega_{1}}$-orbits are

$$
\begin{aligned}
& \mathcal{O}_{0}=\left\{p \in X \mid p \notin \text { line through } p_{0}\right\} \\
& \mathcal{O}_{1}=\left\{p \in X \mid p \in \text { line through } p_{0}, p \neq p_{0}\right\} \\
& \mathcal{O}_{2}=\left\{p_{0}\right\} .
\end{aligned}
$$

We can also describe these orbits as vector bundles over generalized flag varieties

$$
\begin{aligned}
\mathcal{O}_{0} & \rightarrow \mathbb{Q}_{8} \\
\mathcal{O}_{1} & \rightarrow \mathbb{S}_{10} \\
\mathcal{O}_{2} & \rightarrow \text { pt }
\end{aligned}
$$

where $\mathbb{Q}_{8} \cong \mathbb{O P}^{1}$ is the 8-dimensional quadric and $\mathbb{S}_{10} \cong \mathrm{OG}(5,10)$ is the 10-dimensional spinor variety. Indeed, the last fibration is trivial and the second stems from the description of $\mathcal{O}_{1}$ as a cone over $\mathbb{S}_{10}$ (see [8, Lemma 4.1]). Finally, we know from [8] that the Cayley plane also parametrises the family of $\mathbb{Q}_{8}$ 's it contains, hence to $p_{0}$ is associated an 8-dimensional quadric $Q_{0}$. The same goes for $p$, to which corresponds a quadric $Q$. These quadrics are isomorphic to projective octonionic lines $\mathbb{O P} \mathbb{P}^{1}$, and two general such lines meet in one point in $\mathbb{O P}^{2}$, hence the first fibration. 


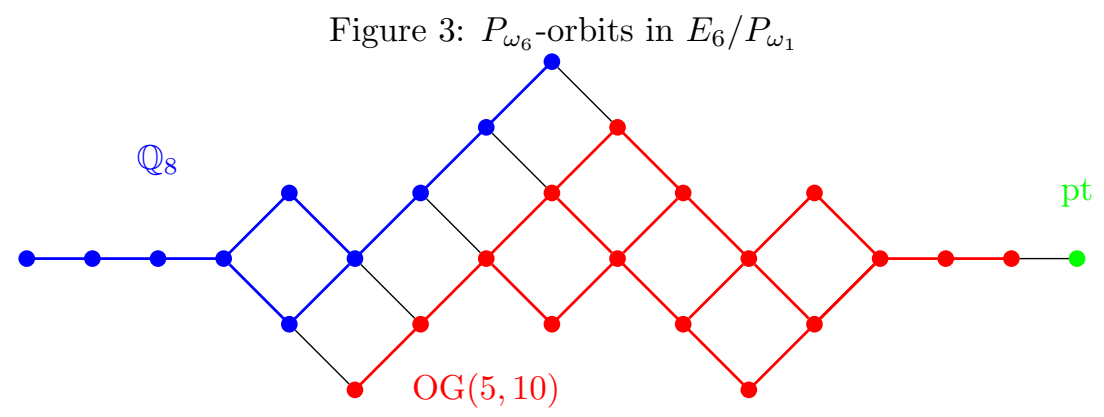

Acknowledgements :

This paper reports on work done during my thesis. I would like to thank my advisor, Laurent Manivel, for his support and for valuable discussions. I am also indebted to Nicolas Perrin for many useful remarks and comments.

\section{References}

[1] Bourbaki, N.: Eléments de mathématiques, fasc. 34: groupes et algèbres de Lie, chap. 4, 5 et 6. Hermann (1968)

[2] Chaput, P.E., Manivel, L., Perrin, N.: Quantum cohomology of minuscule homogeneous spaces. II. Hidden symmetries. Int. Math. Res. Not. IMRN (22), Art. ID rnm107, 29 (2007)

[3] Chaput, P.E., Manivel, L., Perrin, N.: Affine symmetries of the equivariant quantum cohomology ring of rational homogeneous spaces. Math. Res. Lett. 16(1), 7-21 (2009)

[4] Fulton, W.: Intersection Theory. Springer-Verlag, Berlin (1984)

[5] Fulton, W., Pandharipande, R.: Notes on stable maps and quantum cohomology, Algebraic geometrySanta Cruz 1995. In: Proc. Sympos. Pure Math, vol. 62, pp. 45-96 (1997)

[6] Garibaldi, R.: Structurable algebras and groups of type E6 and E7. Journal of Algebra 236(2), 651-691 (2001)

[7] Garsia, A. M., Stanton, D.: Group actions of Stanley-Reisner rings and invariants of permutation groups. Adv. in Math. 51(2), 107-201 (1984)

[8] Iliev, A., Manivel, L.: The Chow ring of the Cayley plane. Compos. Math. 141(1), 146-160 (2005)

[9] Mitchell, S.: Parabolic orbits in flag varieties. preprint 1 (2008)

[10] Perrin, N.: Courbes rationnelles sur les variétés homogènes. Ann. Inst. Fourier (Grenoble) 52(1), 105-132 (2002)

[11] Seidel, P.: $\pi_{1}$ of symplectic automorphism groups and invertibles in quantum homology rings. Geom. Funct. Anal. 7(6), 1046-1095 (1997) 